\title{
An evaluation of image based steganography methods using visual inspection and automated detection techniques
}

\author{
Karen Bailey $\cdot$ Kevin Curran
}

(C) Springer Science + Business Media, LLC 2006

The online article title in the HTML version unfortunately did not include the complete article title. The correct article title is: An evaluation of image based steganography methods using visual inspection and automated detection techniques. The article title appeared correctly in the printed version and in the PDF file.

The online version of the original article can be found at http://dx.doi.org/10.1007/s11042-006-0008-4

K. Bailey $(\square)$

Letterkenny Institute of Technology, Port Road, Letterkenny, Co., Donegal, Ireland e-mail: karen.bailey@lyit.ie

K. Curran

Internet Technologies Research Group, University of Ulster, Magee Campus, Derry, Ireland e-mail: kj.curran@ulster.ac.uk 